\title{
Management of hemoperitoneum due to rupture of the ovarian cyst
}

\author{
Oana Denisa Balalau ${ }^{1,2 *}$, Ileana Maria Conea ${ }^{1,2}$, Nicolae Bacalbasa ${ }^{1}$, Anca Silvia \\ Dumitriu $^{1}$, Stana Paunica ${ }^{1}$, Andrei Vasilache ${ }^{1}$, Octavian Gabriel Olaru ${ }^{1,2}$ \\ ${ }^{1}$ CAROL DAVILA UNIVERSITY OF MEDICINE AND PHARMACY, BUCHAREST, ROMANIA \\ ${ }^{2}$ BUCUR MATERNITY, ST. JOHN CLINICAL EMERGENCY HOSPITAL, DEPARTMENT OF OBSTETRICS AND GYNECOLOGY, BUCHAREST, ROMANIA
}

Ovarian cyst is the most common female gynecological pathology and it is characteristic of reproductive age. Its rupture causes the sudden onset of pelvic-abdominal pain, often associated with physical exertion or sexual contact. The differential diagnosis is made with other causes of lower abdominal pain: ectopic pregnancy, adnexal torsion, pelvic inflammatory disease or acute appendicitis. The clinical picture may vary depending on the type of ruptured cyst. Dermoid cyst causes severe symptoms due to chemical peritonitis that occurs in response to extravasation of sebaceous contents in the peritoneal cavity. Surgical treatment is indicated for complicated forms of cystic rupture. Most cases have self-limiting, quantitatively reduced bleeding and spontaneous resorption within a few days. Patients diagnosed with ovarian cyst are recommended for regular ultrasound monitoring to prevent complications such as cystic rupture or adnexal torsion. The identification of any ovarian tumor mass in the woman at menopause requires further investigation to rule out the causes of malignancy.
\end{abstract}

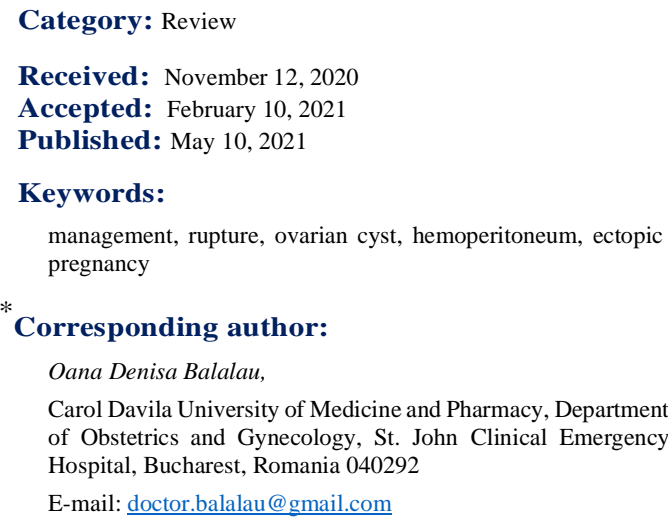

\section{Introduction}

Hemoperitoneum caused by gynecological pathologies is characteristic of young women of childbearing age. The main causes are rupture of ovarian cysts or ectopic pregnancy. Rupture of the ovarian cyst results in the release of cystic fluid or blood that can irritate the peritoneal cavity. Dermoid cysts contain sebaceous fluid, hair, fat, bones or cartilage, and rupture of these cysts results in severe peritoneal irritation. Surgical management is usually required in the event of a ruptured dermoid cyst [1].

Most patients with ruptured ovarian cyst can be treated conservatively by symptomatic medication (analgesic, anti-inflammatory), bed rest, application of an ice pack on the abdomen and continuous clinical and biohumoral monitoring. Only some cases require surgery. Management decisions are based on the characteristics of the case, including the severity of symptoms, the presence of active bleeding, hemodynamic instability and presumptive histological diagnosis [2].

Ovarian cyst rupture occurs more frequently in women of childbearing potential and very rarely in menopausal women. A study performed on 70 patients established that the average age of onset of this pathology is 27 years. The clinical symptoms of the ruptured cyst may vary from no symptoms to sudden appearance of pain located in the lower abdominal quadrant and accentuated unilaterally on the side with the cyst $[1,3]$.

Most often it is located in the right ovary, which is possible due to its position in the vicinity of the sigmoid colon, which provides protection against abdominal trauma. An analysis performed on a group of 244 patients found that $63 \%$ of them were located in the right ovary. The intensity of abdominal pain may be sharp, severe at onset or moderate and progressively accentuated, localized or irradiated. Rupture of the ovarian cyst occurs secondary to physical exertion or sexual contact. When the hemorrhage is severe, the pain radiates to the upper abdominal floor and shoulder due to subphrenic extravasation of the blood $[2,4]$.

The pain in the rupture of the ovarian cyst is due to the irritating effect of the blood. If the intracystic hemorrhage extends to the ovarian cortex, the pain will be focal. When it flows into the peritoneal cavity, it causes irritation of the 
visceral peritoneum and causes radiating pain. The release of intra-abdominal serous or mucinous contents does not cause irritation, even if a significant volume of fluid accumulates intraperitoneally. The rupture of a dermoid cyst and the release of sebaceous fluid produces a marked granulomatous reaction that causes the onset of chemical peritonitis, followed by significant pain [3-5].

\section{Discussions}

For the treatment of hemoperitoneum it is important to establish its cause. The differential diagnosis is made with ectopic pregnancy, hemorrhage secondary to cystic rupture, appendix torsion and acute appendicitis. All of these conditions are associated with significant and sudden onset of the pelvic pain $[1,4]$.

\section{Ectopic pregnancy}

Ectopic pregnancy is localized in $96 \%$ of cases in the fallopian tube. Other locations include: cervical, corneal or interstitial pregnancy, uterine scarr, intramural, ovarian and abdominal [5]. The diagnosis is made based on imaging examination and blood dosing of human chorionic gonadotropin (HCG). Hemoperitoneum in ectopic pregnancy is treated surgically by open or laparoscopic abdominal approach. Surgical options are salpingectomy or salpingostomy, both with similar results on fertility [6].

\section{Adnexal torsion}

Another cause of pelvic-abdominal pain, which makes the differential diagnosis with hemoperitoneum difficult, is adnexal torsion. This requires emergency surgery to avoid compromising the function of the ovary or fallopian tube. Nausea and vomiting often accompany torsion and are less common when the cyst ruptures. When a cyst is involved in the torsion and the patient has pain associated with the presence of intraperitoneal fluid, the distinction between ruptured cyst and torsion is difficult. The difficulty of diagnosis is also demonstrated by a study of 115 cases of adnexal torsion, in which the preoperative diagnosis was established correctly in only $38 \%$ of patients [7].

\section{Pelvic inflammatory disease (PID)}

Pelvic-abdominal pain in young patients, which begins during or shortly after menstruation, with gradual and predominantly bilateral localization is frequently associated with pelvic inflammatory disease (PID). This causes accumulation of free intraperitoneal fluid, mimicking the clinical symptoms of hemoperitoneum. Imaging evaluation guides the clinician on the differential diagnosis [8-10]. Tubo-ovarian abscess is the most severe clinical form of PID. Its rupture is associated with sudden pain and requires emergency treatment to prevent sepsis [11].

\section{Other gynecological conditions}

Other gynecological problems associated with pelvic pain are: endometriosis, leiomyomas, endometritis and Mittelschmerz (ovarian pain). Ovarian hyperstimulation syndrome (OHSS) is an iatrogenic complication of ovulation induction therapy and may be accompanied or confused with cyst rupture. The rupture of large ovarian cysts, secondary to OHSS, is accompanied by ascites and sometimes by pleural and / or pericardial effusion causing electrolyte imbalances (hyponatremia, hyperkalemia), hypovolemia and hypovolemic shock [12].

\section{Other non-gynecological pathologies}

Acute appendicitis is the most common pathology of young adults involved into causing pain in the lower abdominal floor. The duration and nature of pain in appendicitis are different from hemoperitoneum. Classically the pain is sharp, with periumbilical onset, later being located in the right lower quadrant. It is often accompanied by nausea, anorexia and fever [8].

Other non-gynecological pathologies that can cause hemoperitoneum-like pain due to gynecology causes are: nephrolithiasis, interstitial cystitis, irritable bowel syndrome, inflammatory bowel disease and colonic diverticulitis [13,14]. Hemoperitoneum can also be linked to a hepatic, splenic, vascular or coagulopathic etiology [15]. These are often due to a blunt or penetrating traumatic injury to a visceral organ (especially the liver or spleen) or to a blood vessel, or they can be spontaneous.

\section{Treatment of hemoperitoneum of ovarian cystic cause}

Once the differential diagnosis is made, the ovarian cystic hemoperitoneum can be treated conservatively or surgically. Rupture and hemorrhage of an ovarian cyst can be physiological, self-limiting events [16]. They can cause significant hemoperitoneum and require surgery [17]. Due to these diverse clinical situations, there is no wellestablished standard protocol for the management of ruptured ovarian cysts with hemoperitoneum.

In the past, the ovarian cyst ruptured with hemoperitoneum was a surgical emergency. Previous studies have reported the rate of surgery in this situation up to $80 \%[1,18]$. Imaging advances have allowed clinicians to make early diagnosis and therefore to apply appropriate management to patients. Conservative management strategies are alternatives to conventional surgery [19].

CT evaluation of the volume of the hemoperitoneum involves quantifying the amount of pelvic fluid in relation to the distance between the tubal isthmus and the axial plane of the uterus. The bottoms pouches, anterior (ACDS) and posterior (PCDS), are approximated by measuring the distance between the anterior parietal peritoneum and the anterior uterine wall, respectively the posterior and rectum uterine wall. The total volume of hemoperitoneum is the sum of ACDS and PCDS measurements [20,21]. 
Patient monitoring is a good alternative for uncomplicated cases where bleeding is self-limiting. Cases of complicated ruptured ovarian cyst involve the identification of the following signs:

- hemodynamic instability: hypotension, tachycardia

- sensitivity and signs of peritoneal irritation: active and/ or abundant bleeding shown by imaging; patients with a large amount of intraperitoneal blood will show vital signs modification and severe anemia

- signs of an infectious process: fever, leukocytosis and abdominal tenderness

- malignancy suggestive findings: menopausal patients with ovarian tumor.

Conservative treatment is the preferred management protocol for patients with uncomplicated ruptured ovarian cyst, especially in women without coagulopathies. It is recommended to be hospitalized for careful monitoring of vital signs, hematocrit and repeated imaging evaluation to monitor the nature of bleeding (self-limiting or not). The main indications for surgery are: unstable vital signs, a significant decrease in hemoglobin, increased volume of hemoperitoneum (imaging) and severe or persistent abdominal pain, despite the use of analgesics. The decision to perform surgery is at the discretion of the attending physician [16,19].

\section{Surgical treatment}

Ovarian cyst complicated with massive hemorrhage or active bleeding requires hospitalization and surgery. Vital signs will be closely monitored, repeated hematocrit checks and continuous imaging of intraperitoneal fluid will be performed [22].

In a study of 78 women diagnosed with $\mathrm{CT}$ with a broken ovarian cyst and hemoperitoneum, 63 patients were treated conservatively $(80.8 \%)$ and 15 required surgery (19.2\%) [23]. All patients who required surgery were treated laparoscopically, the main cause of hemoperitoneum being the hemorrhagic corpus luteum. In the same study, patients who required surgery showed a faster decrease in hemoglobin within 4 hours $(1.6 \pm 0.6$ versus $1.2 \pm 0.8 \mathrm{~g} / \mathrm{dL}, \mathrm{p}=0.026)$ and needed transfusions more frequently than those treated conservatively $(53.3 \%$ vs. $11.1 \%, \mathrm{p}=0.001)$. The duration of hospitalization was longer in patients with surgery than those who were treated conservatively $(4.1 \pm 0.8$ vs. $3.1 \pm 1.4, p=0.002)$ [23].

Ho et al. reported that in the $1980 \mathrm{~s}$ all patients diagnosed with hemoperitoneum secondary to ovarian cystic rupture were treated surgically. In the 2000s, women with the same pathology received conservative/ medical treatment in only $18.7 \%$ of cases [18]. Although there was an increasing trend of conservative treatment from the 1980 s to the 2000 s, surgery remained the main basis of treatment.
A study by Raziel et al. published in 1993 reported that the surgery rate was $83 \%$ in patients with rupture of the corpus luteum [1]. They emphasized the value of ultrasound and diagnostic laparoscopy in management decisions. $25.7 \%$ of patients required diagnostic laparoscopy without other surgical procedures. 18 patients in Raziel's study had at the same time ectopic pregnancy and contributed to the increase in the rate of surgery [3].

Laparoscopy is the preferred surgical approach because it results in lower morbidity than laparotomy [24]. If laparoscopic equipment is not immediately available or if the surgeon is not trained in laparoscopy, an open laparotomy will be performed.

The value of diagnostic laparoscopy is considered of great significance in establishing the diagnosis when we face equivocal or uncertain conditions.

In the actual study by Jee $H$. et al. surgery was considered only when patients showed unstable vital signs, a significant decrease in hemoglobin or an increase in hemoperitoneum (imaging) or severe or persistent abdominal pain [23]. The above indications are similar to those suggested by Bottomley: hemodynamic imbalance, uncertain diagnosis or suspicion of adnexal torsion, lack of improvement in symptoms within 48 hours of presentation and increase in suspected hemoperitoneum imagistically or by decreased hemoglobin concentration [19,25].

Rupture of dermoid cyst with discharge of sebaceous material into the abdominal cavity is rare, but has serious consequences. Shock and hemorrhage follow up the rupture, which can later develop a marked granulomatous reaction (chemical peritonitis) and the formation of dense adhesions and chronic pain [26]. In this situation, emergency surgery is recommended, which will include aspiration of the cystic contents, abundend lavage with warm saline solution (to avoid hypothermia), aspiration to remove any residual sebaceous material and cystectomy.

The rupture of an endometriotic cyst is associated with significant bleeding [27,28]. Diagnosis and treatment should be established quickly due to the risk of hemodynamic imbalance.

The relatively low rate of surgery is currently explained by the frequent use of imaging assessments, including CT, which allow differential diagnosis with other causes of acute surgical abdomen. A rupture of the corpus luteum with hemoperitoneum was frequently misdiagnosed preoperatively as appendicitis, ectopic pregnancy, endometriosis, and neoplasm when CT was not frequently used [29].

There are no known methods to prevent the rupture of an existing ovarian cyst, except for surgical drainage or removal of the cyst, but hormonal treatments that suppress ovulation can decrease the incidence of new cysts. 


\section{Conclusions}

Rupture of an ovarian cyst is the most common cause of gynecologic hemoperitoneum. Normal ovulation can lead to fluid accumulation in the pelvic cavity, which can be ultrasound misinterpreted as hemoperitoneum. Differential diagnosis is important for establishing therapeutic behavior and can be made with other gynecological or non-gynecological causes of pelvicabdominal pain.

Most patients with ruptured ovarian cyst and hemoperitoneum can be managed conservatively. Hemodynamic instability and a large volume of hemoperitoneum detected through imaging require surgery. Initial hemoglobin levels are not helpful in anticipation of emergency surgery, which may be explained by the fact that the initial hematocrit may not reflect acute blood loss.

CT completes the ultrasonographic evaluation, having the advantage of evaluating the source and amount of bleeding. Laparoscopy is the first-line approach, open laparotomy being reserved for particular cases. The presence of an adnexal tumor mass in the menopausal woman should raise the suspicion of malignancy and require further investigation to establish the diagnosis.

\section{Conflict of interest disclosure}

There are no known conflicts of interest in the publication of this article. The manuscript was read and approved by all authors.

\section{Compliance with ethical standards}

Any aspect of the work covered in this manuscript has been conducted with the ethical approval of all relevant bodies and that such approvals are acknowledged within the manuscript.

\section{References}

1. Raziel A, Ron-El R, Pansky M, Arieli S, Bukovsky I, Caspi E. Current management of ruptured corpus luteum. Eur J Obstet Gynecol Reprod Biol. 1993; 50(1):77-81. doi: 10.1016/0028-2243(93)90168-c. PMID: 8365540.

2. Kim JH, Lee SM, Lee JH, Jo YR, Moon MH, Shin J, Kim BJ, Hwang KR, Lee TS, Bai KB, Jeon HW. Successful conservative management of ruptured ovarian cysts with hemoperitoneum in healthy women. PLoS One. 2014;9(3):e91171. doi: 10.1371/journal.pone.0091171

3. Abduljabbar HS, Bukhari YA, Al Hachim EG, Alshour GS, Amer AA, Shaikhoon MM, Khojah MI. Review of 244 cases of ovarian cysts. Saudi Med J. 2015; 36(7):834-8. doi: 10.15537/smj.2015.7.11690
4. Patel MD, Feldstein VA, Lipson SD, Chen DC, Filly RA. Cystic teratomas of the ovary: diagnostic value of sonography. AJR Am J Roentgenol. 1998;171(4):10615. doi: 10.2214/ajr.171.4.9762997

5. Bouyer J, Coste J, Fernandez H, Pouly JL, Job-Spira N. Sites of ectopic pregnancy: a 10 year population-based study of 1800 cases. Hum Reprod. 2002;17(12):322430. doi: 10.1093/humrep/17.12.3224

6. Hajenius PJ, Mol F, Mol BW, Bossuyt PM, Ankum WM, van der Veen F. Interventions for tubal ectopic pregnancy. Cochrane Database Syst Rev. 2007;2007(1):CD000324. doi: 10.1002/14651858.CD000324.pub2

7. Argenta PA, Yeagley TJ, Ott G, Sondheimer SJ. Torsion of the uterine adnexa. Pathologic correlations and current management trends. J Reprod Med. 2000 Oct;45(10):831-6.

8. Lane R, Grabham J. A useful sign for the diagnosis of peritoneal irritation in the right iliac fossa. Ann $R$ Coll Surg Engl. 1997;79(2):128-9.

9. Nishino M, Hayakawa K, Iwasaku K, Takasu K. Magnetic resonance imaging findings in gynecologic emergencies. J Comput Assist Tomogr. 2003;27(4): 564-70. doi: 10.1097/00004728-200307000-00021

10. Bennett GL, Slywotzky CM, Giovanniello G. Gynecologic causes of acute pelvic pain: spectrum of CT findings. Radiographics. 2002;22(4):785-801. doi: 10.1148/radiographics.22.4.g02j118785

11. Pedowitz P, Bloomfield RD. Ruptured adnexal abscess (tuboovarian) with generalized peritonitis. Am J Obstet Gynecol. 1964;88:721-9. doi: 10.1016/0002-9378(64)90604-0

12. Golan A, Ron-el R, Herman A, Soffer Y, Weinraub Z, Caspi E. Ovarian hyperstimulation syndrome: an update review. Obstet Gynecol Surv. 1989;44(6):43040. doi: 10.1097/00006254-198906000-00004

13. Chapman WP, Herrera R, Jones CM. A comparison of pain produced experimentally in lower esophagus, common bile duct, and upper small intestine with pain experienced by patients with diseases of biliary tract and pancreas. Surg Gynecol Obstet. 1949;89(5):57382 .

14. Brown FR. The Problem of Abdominal Pain. Br Med J. 1942;1(4243):543-6. doi: 10.1136/bmj.1.4243.543

15. Lucey BC, Varghese JC, Anderson SW, Soto JA. Spontaneous hemoperitoneum: a bloody mess. Emerg Radiol. 2007;14(2):65-75. doi: 10.1007/s10140-0070594-0

16. Queenan JT, O'Brien GD, Bains LM, Simpson J, Collins WP, Campbell S. Ultrasound scanning of ovaries to detect ovulation in women. Fertil Steril. 1980;34(2):99-105.

17. Hertzberg BS, Kliewer MA, Paulson EK. Ovarian cyst rupture causing hemoperitoneum: imaging features and the potential for misdiagnosis. Abdom Imaging. 1999; 24(3):304-8. doi: 10.1007/s002619900502 
18. Ho WK, Wang YF, Wu HH, Tsai HD, Chen TH, Chen M. Ruptured corpus luteum with hemoperitoneum: case characteristics and demographic changes over time. Taiwan J Obstet Gynecol. 2009;48(2):108-12. doi: 10.1016/S1028-4559(09)60267-9

19. Bottomley C, Bourne T. Diagnosis and management of ovarian cyst accidents. Best Pract Res Clin Obstet Gynaecol. 2009;23(5):711-24. doi: 10.1016/j.bpobgyn.2009.02.001

20. Lucey BC, Varghese JC, Anderson SW, Soto JA. Spontaneous hemoperitoneum: a bloody mess. Emerg Radiol. 2007;14(2):65-75. doi: 10.1007/s10140-0070594-0

21. Lucey BC, Varghese JC, Soto JA. Spontaneous hemoperitoneum: causes and significance. Curr Probl Diagn Radiol. 2005;34(5):182-95. doi: 10.1067/j.cpradiol.2005.06.001

22. Gupta A, Gupta S, Manaktala U, Gupta MM, Solanki V. Conservative management of corpus luteum haemorrhage in patients on anticoagulation: a report of three cases and review of literature. Arch Gynecol Obstet. 2015;291(2):427-31. doi: 10.1007/s00404-0143394-2

23. Kim JH, Lee SM, Lee JH, Jo YR, Moon MH, Shin J, Kim BJ, Hwang KR, Lee TS, Bai KB, Jeon HW. Successful conservative management of ruptured ovarian cysts with hemoperitoneum in healthy women. PLoS One. 2014;9(3):e91171. doi: 10.1371/journal.pone.0091171
24. Teng SW, Tseng JY, Chang CK, Li CT, Chen YJ, Wang PH. Comparison of laparoscopy and laparotomy in managing hemodynamically stable patients with ruptured corpus luteum with hemoperitoneum. J Am Assoc Gynecol Laparosc. 2003;10(4):474-7. doi: 10.1016/s1074-3804(05)60147-8

25. Thorson CM, Ryan ML, Van Haren RM, Pereira R, Olloqui J, Otero CA, Schulman CI, Livingstone AS, Proctor KG. Change in hematocrit during trauma assessment predicts bleeding even with ongoing fluid resuscitation. Am Surg. 2013;79(4):398-406.

26. Koshiba H. Severe chemical peritonitis caused by spontaneous rupture of an ovarian mature cystic teratoma: a case report. J Reprod Med. 2007; 52(10):965-7.

27. Togami S, Kobayashi H, Haruyama M, Orita Y, Kamio M, Douchi T. A very rare case of endometriosis presenting with massive hemoperitoneum. J Minim Invasive Gynecol. 2015;22(4):691-3. doi: 10.1016/j.jmig.2015.02.014

28. Tsai HJ. Suitable timing of surgical intervention for ruptured ovarian endometrioma. Taiwan J Obstet Gynecol. 2015;54(1):105. doi: 10.1016/j.tjog.2014.11.019

29. Payne JH, Maclean RM, Hampton KK, Baxter AJ, Makris M. Haemoperitoneum associated with ovulation in women with bleeding disorders: the case for conservative management and the role of the contraceptive pill. Haemophilia. 2007;13(1):93-7. doi: 10.1111/j.1365-2516.2006.01399.x 